FACTA UNIVERSITATIS

Series: Mechanical Engineering Vol. 18, No 1, 2020, pp. 57 - 68

https://doi.org/10.22190/FUME171128002S

Original scientific paper

\title{
NUMERICAL INVESTIGATION OF THE INFLUENCE OF THE DOUBLY CURVED BLADE PROFILES ON THE REVERSIBLE AXIAL FAN CHARACTERISTICS
}

\author{
Živan Spasić, Miloš Jovanović, Jasmina Bogdanović-Jovanović, \\ Saša Milanović
}

Faculty of Mechanical Engineering, University of Niš, Serbia

\begin{abstract}
In reversible axial fans a change in the direction of the impeller rotation is accompanied with a change in the direction of the working fluid flow. To satisfy the flow reversibility, the impeller blades are usually designed with straight symmetrical profiles. The flow reversibility may also be achieved by using asymmetrical blade profiles in which, to satisfy the equality of the leading and trailing angle of the profiles, the mean line of the profile has to have a double curvature in the shape of the stretched letter ' $S$ '. The paper numerically investigates the influence of the doubly curved blade profiles on the reversible axial fan characteristics. Numerical simulations are carried out on an axial fan only with the impeller, with the blades that have double-curved mean line profiles for different values of the angles at the profile ends. For numerical simulation the ANSYS CFX software package is used. Results of the numerical simulation are shown in diagrams $\Delta p(Q), \eta(Q)$ and $P(Q)$ at different angles of the profile ends. On the basis of the simulation and analysis of the characteristics, appropriate conclusions are proposed, along with the most advantageous profile of the blades.
\end{abstract}

Key Words: Reversible Axial Fan, Curved Profile, Angle, Characteristics, Numerical Simulations

\section{INTRODUCTION}

Reversible axial fans are used to achieve a forced air-gas flow in the primary and reverse flow regime. The fans only have a single impeller whose reversibility of the flow is achieved by changing the direction of rotation $[1,2,3]$. To satisfy the reversibility of the flow, with the identical curve performance for both regimes, the impeller blades are

Received November 28, 2017 / Accepted January 12, 2018

Corresponding author: Živan Spasić

Faculty of Mechanical Engineering, University of Niš, Aleksandra Medvedeva 14, 18000 Niš, Serbia

E-mail:zivans@masfak.ni.ac.rs 
usually designed with straight symmetrical profiles. Efficiency of these fans is relatively low due to the large incidence angle flow on the profile of the blades $[4,5]$.

Classical axial fans, designed for one direction of flow, can work as reversible fans as well. The characteristics of such fans in a reversible regime are worse due to unfavorable flow conditions. The fan characteristics are less different in the direct and reversible mode with a fan with a smaller curvature of the blade profile $[2,3]$.

The efficiency of the fan depends on the shapes of the blade profiles, the blade itself, and the ratio of the diameters of the impeller hub and the shroud [2, 6]. Numerical flow simulations in fans can determine the best shape profiles for achieving better efficiency of the fans, which can serve for further experimental tests $[7,8,9]$. Various commercial programs are available for numerical simulations. The k- $\varepsilon$ model, employed in this study, is frequently used as the method for solving the turbulent flow in turbomachinery [9-11].

The reversible axial fan is designed with the blades having a double curved profile for increase pressure [1,12]. This paper presents the original profile design with a slightly double curved mean line. The fan is designed based on the design of the fan blades with straight profiles. To form the profile with a doubly curved mean line, normally the thickness of the straight symmetric profile marked as PP2 is applied [1, 13]. The shape of the profile is determined after a series of numerical simulations of flow in these fans with different angles of the profile end curvature [1]. This paper presents the comparison of the characteristics of the fan with the straight profiles of the blade numerically obtained. We should be careful with the introduction of double curved profiles since any change in the curvature can lead to separation of the flow from the surface of the blade profile and thus to the degradation of the fans' performance $[14,15]$.

\section{THE BASIC GEOMETRY OF THE REVERSIBLE AXIAL FAN}

The basic geometry of the reversible axial fan is designed with the straight profiles of blades (Fig. 1) [1, 13]. In order to achieve equality of fluid energy exchange along the radius of the impeller, the blades of the impeller are spatially curved. The fan impeller is designed under the principle of equal specific work of all elementary stages, that is, under the principle of the flow along axially symmetrical cylindrical surfaces $[3,6,15]$. To determine the shape of the fan blades, the profiles are designed in 13 elementary stages, approximately equally distributed along the height of the blade. The design was performed using the method of lift force for straight profiles [1, 13]. In a cascade with straight profiles the leading and trailing angle of blade profile $\left(\beta_{1 l}=\beta_{2 l}\right)$ equals the angle of inclination profile $\beta_{t}$ in the cascade profile (Fig. 1):

$$
\beta_{1 l}=\beta_{2 l}=\beta_{t}
$$

Fig. 1 schematically shows a meridian section of the reversible axial fan, with a developed cylindrical section for the mean section of the impeller. The straight profiles are set to step $t$, the angle of inclination $\beta_{t}$ profile that changes the height of the blade. The primary flow of the fluid is left-to-right flow. In Fig. 1 the primary flow is indicated by speed $\vec{c}$ corresponding to the direction of circumferential speed $\vec{u}$. With the change in the flow direction (circumferential velocity direction $-\vec{u}$ ), the flow becomes reversible (flow velocity $-\vec{c}$ ). 


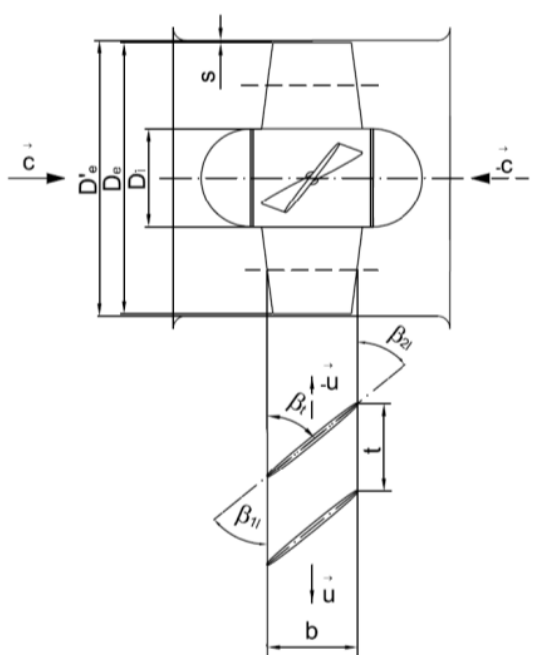

Fig. 1 Reversible axial fan

The diameter of the fan impeller hub and other geometrical values are obtained according to the recommendations for optimal values of the dimensionless volume coefficient and the pressure coefficient. For the calculation parameters of flow, volumetric flow rate $Q=3.61 \mathrm{~m}^{3} / \mathrm{s}$, total pressure increase $\Delta p_{\text {tot }}=180 \mathrm{~Pa}$, rotation speed $n=1405 \mathrm{rpm}$ and assumed efficiency $\eta=0.65$, the basic geometry of the fan impeller is obtained $[1,13]$ :

- $D_{\mathrm{i}}=300 \mathrm{~mm}$ - diameter of the fan impeller hub,

- $D_{\mathrm{e}}=630 \mathrm{~mm}$ - peripheral diameter of the fan impeller, and,

- $z_{\mathrm{K}}=6$ - number of impeller blades.

\section{Profiles with a Doubly Curved Mean Line - Asymmetric Profiles}

The flow reversibility at the reversible axial fan can be achieved by applying blades with asymmetrical profiles, which have a doubly curved profile shape mean line in the shape of a stretched 'S' (Fig. 2). In order to achieve the same characteristics for both directions of flow, the leading and trailing angles of the profile blades should be equal $\beta_{1 l}$ $=\beta_{2 l}$. They are different from the angle of inclination profile $\beta_{t}$ in the cascade profile $\beta_{1 l}$ $=\beta_{2 l} \neq \beta_{t}$ depending on the angles of the curvature of the ends of profile $\Delta \beta_{l}$ (Fig. 4). For the purpose of creating a profile, the thickness of a straight symmetric profile PP2 is usually applied to the mean line $[1,13]$.

\subsection{Mean line design in the profiles with a double curvature}

The design shape of the mean line of this profile is determined after a series of numerical simulations of flow in the straight profile cascades [12].

Fig. 2 shows the design of the mean line of the profile with a double curvature: rays $\left(\mathrm{a}_{1}\right.$ and $\mathrm{a}_{2}$ ) are drawn from the ends of the profile (point $A_{1}$ and $A_{2}$ ), under the chosen angle of curvature of profile mean line $\Delta \beta_{l}$. The rays with verticals, drawn along length $l_{1}$ 
$\left(l_{1}=0,2 \cdot l\right)$ of the ends of the profile, make intersection points $B_{1}$ and $B_{2}$. The line joining points $\left(B_{1}\right.$ and $\left.B_{2}\right)$ passes through the middle mean line of the straight profile, point $\mathrm{O}$. Curvature of the profile is obtained by connecting the lines $\left(A_{1} B_{1}\right.$ and $B_{1} B_{2}$ on the one hand, and $\overline{A_{2} B_{2}}$ and $\overline{B_{2} B_{1}}$ on the other) with radius $R(R=l)$. The perpendicular distance between the rays $\left(\mathrm{a}_{1}\right.$ and $\mathrm{a}_{2}$ ) drawn from the end points (point $A_{1}$ and $A_{2}$ ) is marked with $e$ in Fig. 2. Relative size $\bar{e}(\bar{e}=e / l)$, in relation to the profile length, with the angle of profile curvature $\Delta \beta_{l}$, is expressed by the relation: $\bar{e}=l \cdot \sin \Delta \beta_{l}$.

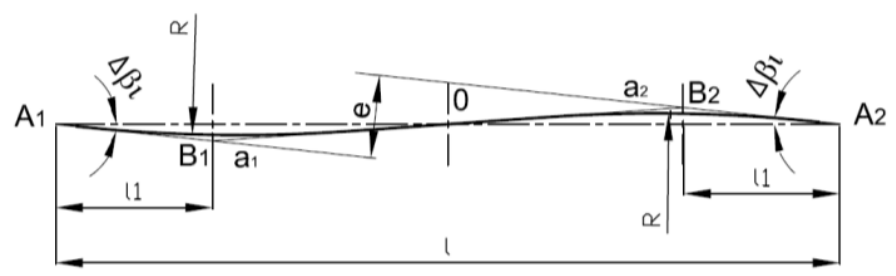

Fig. 2 Design of the mean line of the doubly curved profile

The profiles with a doubly curved mean line have equal angles of curvature of the profile ends, which along with the centerline of the profile $\left(A_{1}-A_{2}\right)$ amounts to $\Delta \beta_{l}$.

\subsection{Designing the curved profile}

The curved profile is designed by perpendicularly applying thickness $\delta_{j}$ of the straight symmetrical profile marked as PP2 $[1,13]$ along the doubly curved mean line $l_{\mathrm{j}}$ for the intersection $j$ (Table 1, Fig. 3).

Table 1 Distribution of thickness along the profile mean line $[1,13]$

\begin{tabular}{lccccccccc}
\hline$\left(l_{j} / l\right) \times 10^{-2}$ & 0.00 & 2.14 & 3.57 & 5.62 & 10.53 & 20.43 & 30.24 & 40.14 & 50.00 \\
$\left(\delta_{j} / \delta_{\max }\right) \times 10^{-2}$ & 0.00 & 43.33 & 50.00 & 58.33 & 73.33 & 86.67 & 95.00 & 96.67 & 100.0 \\
\hline$\left(l_{j} / l\right) \times 10^{-2}$ & 59.77 & 69.58 & 79.48 & 89.38 & 94.29 & 96.52 & 97.86 & 100.0 & \\
$\left(\delta_{j} / \delta_{\max }\right) \times 10^{-2}$ & 96.67 & 95.00 & 86.67 & 73.33 & 58.33 & 50.00 & 43.33 & 0.00 & \\
\hline
\end{tabular}

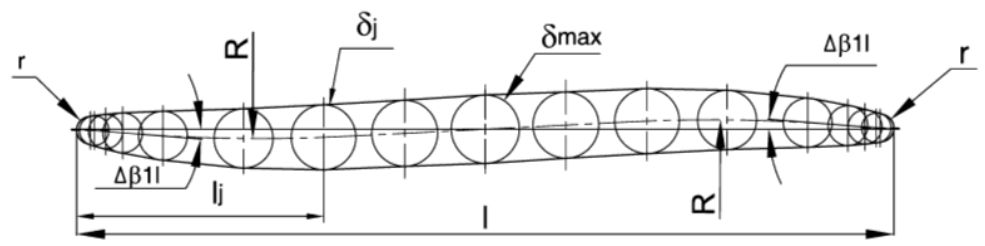

Fig. 3 Geometry of the doubly curved profile

$l$ - the profile length $\delta_{\text {max }}-$ the maximum profile thickness, $r$ - the radius of the curvature of the profile ends, $R$ - the radius of the profile curvature, $\Delta \beta l$ - the angle of the curvature of the profile ends 
If one observes a cascade with profiles that have a doubly curved mean line, the leading and trailing angles of blade profile $\left(\beta_{1 l}=\beta_{2 l}\right)$ are greater or smaller than the angle of inclination of profile cascade $\beta_{t}$, for the angle of end curvature of profile mean line $\Delta \beta_{l}$ (Fig. 4):

$$
\beta_{1 l}=\beta_{2 l}=\beta_{t} \pm \Delta \beta_{l}
$$

Fig. 4 shows the position of the doubly curved profiles in the cascade profile with $\beta_{1 l}=\beta_{2 l}$ $>\beta_{t}$ (Fig. 4-a) and $\beta_{1 l}=\beta_{2 l}<\beta_{t}$ (Fig. 4-b).

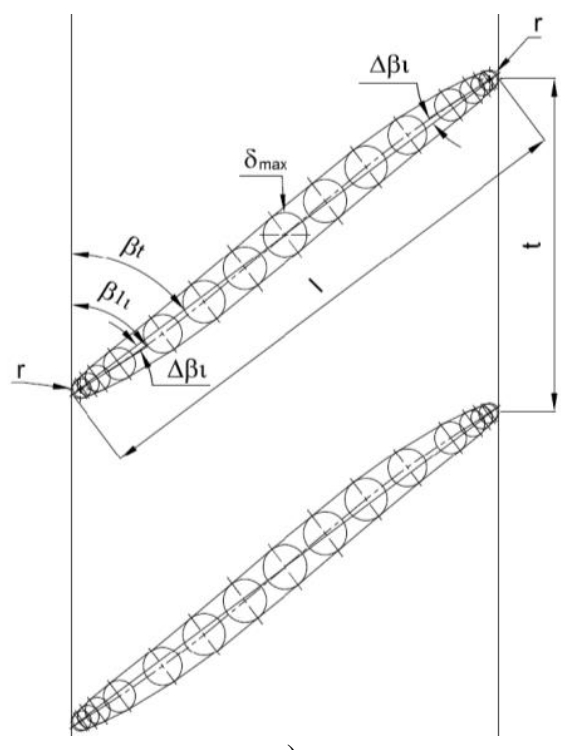

a)

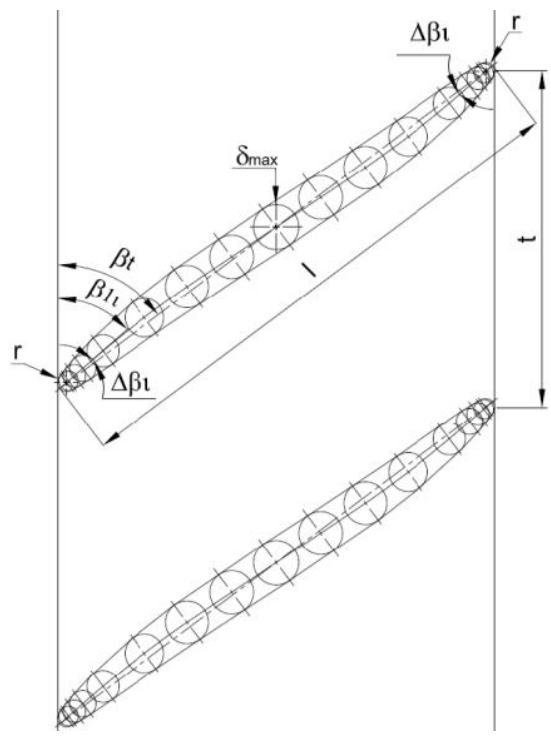

b)

Fig. 4 Cascades with doubly curved profiles: a) Leading and trailing angles of the profile $\beta_{1 l}=\beta_{2 l}=\beta_{t}+\Delta \beta_{l},\left(\beta_{1 l}<\beta_{t}\right)$, b) Leading and trailing angles of the profile $\beta_{1 l}=\beta_{2 l}=\beta_{t^{-}}$ $\Delta \beta_{l},\left(\beta_{1 l}>\beta_{t}\right)$

\section{NUMERICAL SimUlATION OF FLOW IN THE REVERSIBLE AXIAL FAN}

In order to determine the best profile shape, numerical simulations are carried out for the flow in an reversible axial fan with the blades which possess different angles of curvature of the profile mean line ends: $\Delta \beta_{l}= \pm 2.9^{0}(\bar{e}=0.05), \Delta \beta_{l}= \pm 4.5^{0}(\bar{e}=0.078)$ and $\Delta \beta_{l}= \pm 6^{0}(\bar{e}=0.1)$.

\subsection{Model of the reversible axial fan for numerical simulations}

The blades of the model fan for numerical simulations are formed on the basis of the fan designed with straight profiles for seven cylindrical sections of the impeller $x$ $(x=\mathrm{I}-\mathrm{VII}) \quad$ (seven elementary stages) [1, 13], whose positions are defined by radii $r_{x}$ (Table 2). The profile geometry of radii $r_{x}$ (Table 2, Fig. 5) is defined by profile length $l_{x}$, 
thickness distribution $\delta_{j}$ along the mean line, as shown in Fig. 3, the maximum thickness in the middle of the profile $\delta_{\max , \mathrm{x}}$, the profile leading and tail curvature radii $r_{1 x}=r_{2 x}$ and the angle of profile inclination $\beta_{\mathrm{tx}}$.

Table 2 Geometry of the profile cascade in cylindrical sections

\begin{tabular}{cccccccc}
\hline Section: $x$ & $\begin{array}{c}r_{x} \\
{[\mathrm{~mm}]}\end{array}$ & $\begin{array}{c}t_{x} \\
{[\mathrm{~mm}]}\end{array}$ & $\begin{array}{c}l_{x} \\
{[\mathrm{~mm}]}\end{array}$ & $\begin{array}{c}\beta_{\mathrm{tx}} \\
{\left[{ }^{\circ}\right]}\end{array}$ & $\begin{array}{c}\delta_{\max , \mathrm{x}} \\
{[\mathrm{mm}]}\end{array}$ & $\begin{array}{c}\left(\delta_{\max } / l\right)_{x} \\
{[-]}\end{array}$ & $\begin{array}{c}r_{l x}=r_{2 x} \\
{[\mathrm{~mm}]}\end{array}$ \\
\hline I & 150 & 157 & 144 & 53.7 & 12 & 0.083 & 2.4 \\
II & 178 & 186 & 138 & 42.3 & 11 & 0.080 & 2.2 \\
III & 205 & 215 & 133 & 36.4 & 10 & 0.075 & 2.0 \\
IV & 233 & 243 & 126 & 31.8 & 9 & 0.071 & 1.8 \\
V & 260 & 272 & 121 & 28.5 & 8 & 0.066 & 1.6 \\
VI & 288 & 301 & 114 & 26.0 & 7 & 0.061 & 1.4 \\
VII & 315 & 330 & 108 & 24.6 & 6 & 0.056 & 1.2 \\
\hline
\end{tabular}

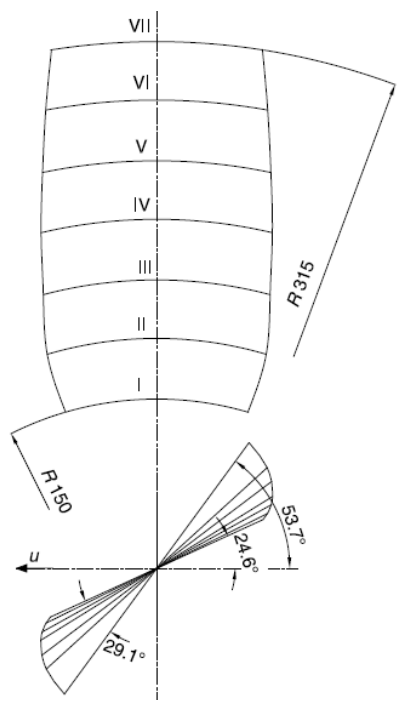

Fig. 5 Blade with the profile mean lines developed in a plane, $\Delta \beta_{\mathrm{L}}=29.1^{\circ}$

The difference in the inclination angles of the profile mean line at the hub $\left(\left(\beta_{\mathrm{t}}=\beta_{\mathrm{tI}}\right.\right.$ $\left.=53.7^{\circ}\right)$ and the shroud $\left(\beta_{\mathrm{te}}=\beta_{\mathrm{tVII}}=24.6^{\circ}\right)$, the angular spatial blade curvature $\Delta \beta_{\mathrm{L}}$ (Fig. 5), is $\Delta \beta_{\mathrm{L}}=\Delta \beta_{\mathrm{t}}=\beta_{\mathrm{ti} .}-\beta_{\mathrm{te} .}=53.7-24.6=29.1^{\circ}$

The blades are mounted on the hub under a specific blade angle $\left(\beta_{\mathrm{ti}}\right)$, which is defined in accordance with the profile inclination angle at the hub $\left(\beta_{\mathrm{L}}=\beta_{\mathrm{ti}}\right)$. The angles of the profile ends for each cylindrical cross section (x) of the impeller are defined in relation to the inclination angle of the profile, i.e., in relation to the inclination angle of the mean line of the straight profiles (the profile PP2, Table 2), as well as $\beta_{1 l}=\beta_{2 l}=\beta_{t} \pm \Delta \beta_{l}$.

The mean lines of the blade profile developed in a plane with angles $\beta_{1 l}=\beta_{2 l}>\beta_{t}$ are shown in Fig. 6-a, and the mean lines of the blade profile developed in a plane with angles $\beta_{1 l}=\beta_{2 l}<\beta_{t}$ are shown in Fig. 6-b. 


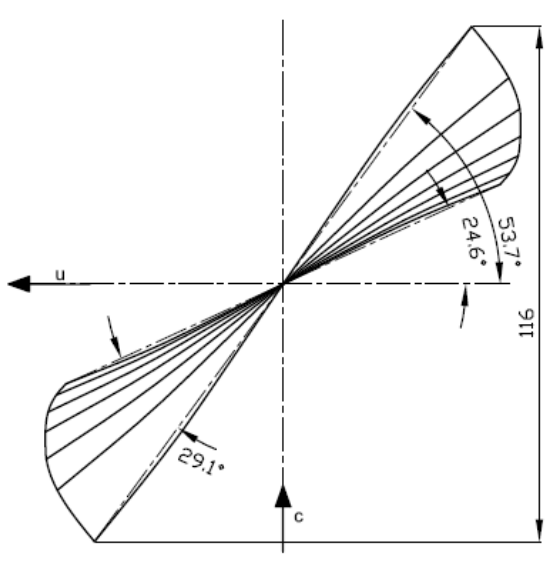

a)

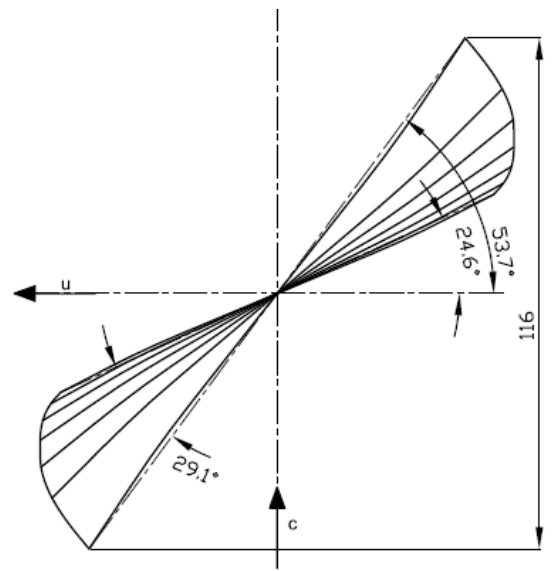

b)

Fig. 6 Mean lines of the blade profile developed in a plane, a) profile with angles $\beta_{1 l}=\beta_{2 l}$ $>\beta_{t}$, b) profile with angles $\beta_{1 l}=\beta_{2 l}<\beta_{t}$

\subsection{Numerical flow simulations in ANSYS CFX}

In order to investigate the influence of the doubly curved blade profiles on the performance of a reversible axial fan, numerical simulations of flow are carried out for the design of a fan with one impeller, with the blades that have doubly curved mean line profiles, for different flow through the fan $Q=(11000 \div 13500) \mathrm{m}^{3} / \mathrm{h}$, impeller speed $n=1405 \mathrm{~min}^{-1}$ and air density $\rho=1.2 \mathrm{~kg} / \mathrm{m}^{3}$ [1]. Numerical simulations of the fan flow can provide aerodynamic characteristics. One of the most popular software packages for turbomachinery flow simulation, ANSYS CFX, is used for the numerical simulation of fluid $[9,11,16]$.

\subsubsection{Model geometry formation}

The model geometry is formed by drawing a 3D model in a specialized part of software for the design of impellers of turbomachinery, ANSYS CFX-BladeGen (Fig. 7-a). Flow space is defined by the borders of entrance and exit from the impeller hub, shroud and blades of the impeller. Blade geometry is defined by several cylindrical sections (elementary stages). In this case, seven cylindrical sections are chosen for the application of the profile geometry (Table 1 and Table 2).

Profile geometry at each intersection is defined by a comprehensive profile angle $\theta_{r}$, profile inclination angle $\beta_{\mathrm{t}}$ in the radial direction or the profile inclination angle in the axial direction $\left(\beta_{a}=90^{0}-\beta_{\mathrm{t}}\right)$, and a thickness profile along the mean line of the profiles with the profile leading and tail curvature radii.

This program has the option to 'compare'; thus it is possible to make a comparison of the profile geometry of a particular section of the impeller blades with a different profile cross-section by overlapping on the same profile (Fig. 7-b). This feature is particularly important when performing simulations to compare the performance of fans with various shapes of the profile. To assess the influence of the shape profile on the performance, it is 
necessary to set up profiles of different forms of the same angle of profile inclination $\beta_{t}$ because the characteristics of the fan are influenced by the inclination angle of the profile in cascade $\beta_{t}$, in addition to effect of the profile shape.

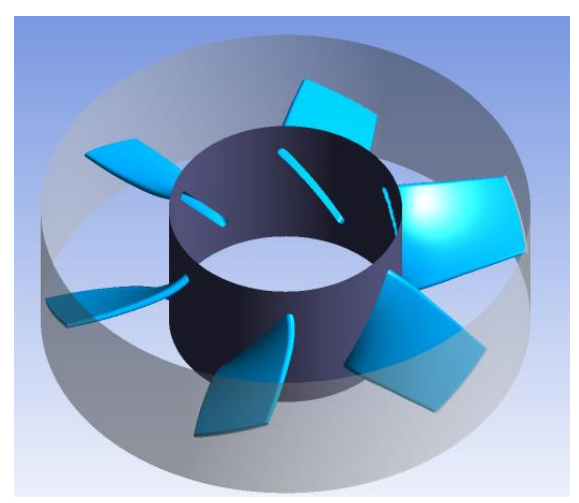

a)

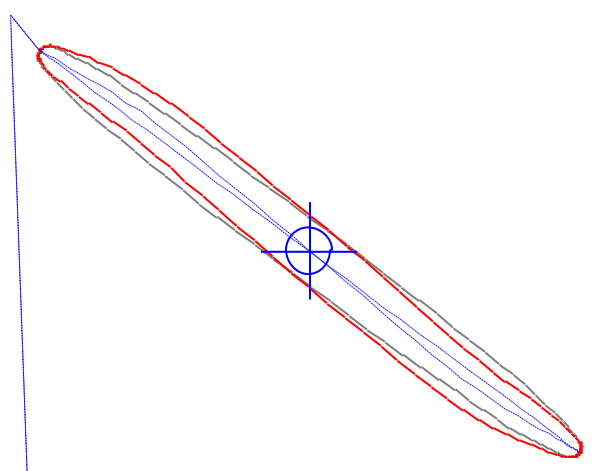

b)

Fig. 7 a) Fan model for the numerical simulation, b) Comparison of the geometry of straight and curved profiles

\subsubsection{Creating the mesh}

On the basis of the defined geometry model, a discretization mesh of the flow field is formed. This is one of the most difficult phases because the quality of the mesh depends on the accuracy of numerical simulation. On the other hand, one cannot ignore the constraints of the computing capacity, namely, those that limit the number of mesh nodes and the size of the mesh elements. When solving this problem a mesh of non-uniform flow field can be created, and it is significantly finer in those areas that are particularly important for research in the defined task. In turbomachinery, because of the symmetry of the impeller, only one of the blades can be considered along with a half of the space between the blades. This fact allows the formation of a finer mesh to shorten the calculation time of numerical simulations.

There are a number of software versions for the creation of a mesh of the model, which in a sense makes it easier to prepare the model for numerical simulation. The mesh is formed in the software for creating a mesh for turbomachinery impellers TurboGrid ANSYS, which is part of the ANSYS software package for simulating flow. In this program, the user first defines the input and output of the simulated domain. The input and output are at a distance of $100 \mathrm{~mm}$ in front of, or behind, the impeller axis perpendicular to the axis of rotation (Fig. 8-a). The radial clearance between the blades of the impeller is 2.5 $\mathrm{mm}$. The number of mesh elements of $1 / 6$ of the fan impeller is about 1000000 for all simulations. The mesh is made up from the topology $(\mathrm{H} / \mathrm{F} / \mathrm{C} / \mathrm{L}$-grid) with a mesh around the profile (O-grid), the mesh setting is performed according to the criteria recommended for maximum and minimum values of the elements: the relation between the edges, volume ratio and angle elements. 


\subsubsection{Defining the physical parameters of flow}

Defining the initial and boundary conditions, fluid characteristics and other physical parameters is done in the pre-processor of ANSYS CFX-Pre. This program defines the physics of the flow process. This determines whether the geometric model and its component parts are at rest or moving. Rotating motion of the impeller is defined as the axis of rotation and rotational speed $\left(n=1405 \mathrm{~min}^{-1}\right)$. Then, each of the interface geometry is assigned with the boundary conditions (input, output, solid surfaces-walls) and the initial value, the total pressure at the entrance $\left(p_{\text {Itot }}=100 \mathrm{kPa}\right)$ and the desired mass fan flow. When the model consists of several domains, it is necessary to define the places of their merger (the interface). Due to the symmetry of the impeller, the simulation is performed only in the space surrounding one blade, so it is necessary to also define the periodic surfaces of the flow field (Fig. 8-b).

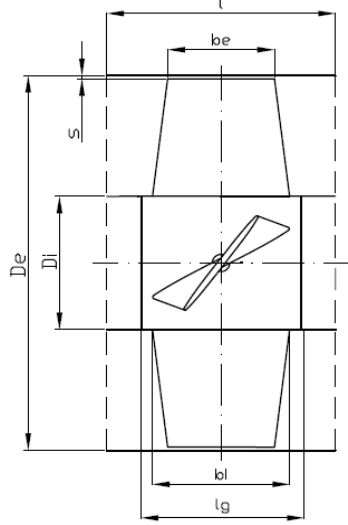

a)

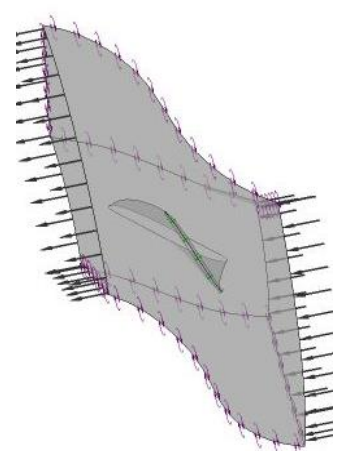

b)

Fig. 8 Appearance of the simulated domain. The marks and values: $D_{\mathrm{i}}=300 \mathrm{~mm}-$ hub diameter, $D_{\mathrm{e}}^{\prime}=635 \mathrm{~mm}-$ shroud diameter, $s-$ tip clearance, $l_{\mathrm{g}}=120 \mathrm{~mm}-$ length of the hub, $b_{\mathrm{i}}=116 \mathrm{~mm}$ - the width of the blade at $D \mathrm{i}, b_{e}=45 \mathrm{~mm}$ - the width of the blade at $D_{e}, l=200 \mathrm{~mm}$ - length of the simulated domain.

This part of the program defines the type of fluid and its physical properties, the turbulence model (in this case, the $k-\varepsilon$ model), and the criteria for numerical calculations (convergence - residual $10^{-5}$, the maximum number of iterations, the level of resolution, etc.).

\subsection{Results of numerical simulation}

The results of numerical simulations are presented in diagrams $\Delta p(Q), \eta(Q)$ and $P(Q)$, which are given on the basis of the averaged values of simulation for the middle cylindrical section of the impeller, for different angles of the blade profile ends. All simulations are carried out for the angle of inclination of the impeller blades (mounting angle of blades) $\beta_{L}=53.7^{0}$, which is measured at the hub and is equal to the angle of inclination of the mean line of the profiles blade around the hub $\left(\beta_{L}=\beta_{t i}\right)$.

In order to assess the influence of the curvature of the blade profile on the performance of the fan, the diagrams also show the characteristics of the fan with the straight profile of the blades (profile PP2), also marked as $\Delta \beta_{l}=0^{0}$ in the diagrams. 


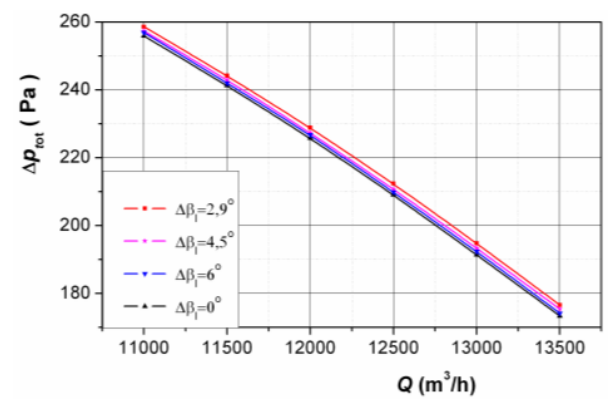

a) $\beta_{1 l}=\beta_{2 l}=\beta_{t}+\Delta \beta_{l}$

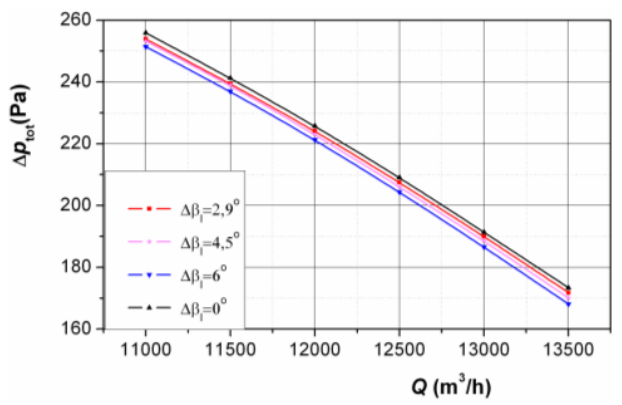

b) $\beta_{1 l}=\beta_{2 l}=\beta_{t}-\Delta \beta_{l}$

Fig. 9 The influence of curvature on the total rise in pressure, $\Delta p_{t o t}(Q)$

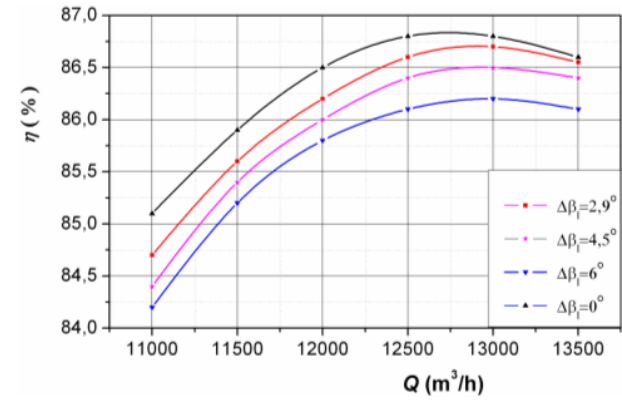

a) $\beta_{1 l}=\beta_{2 l}=\beta_{t}+\Delta \beta_{l}$

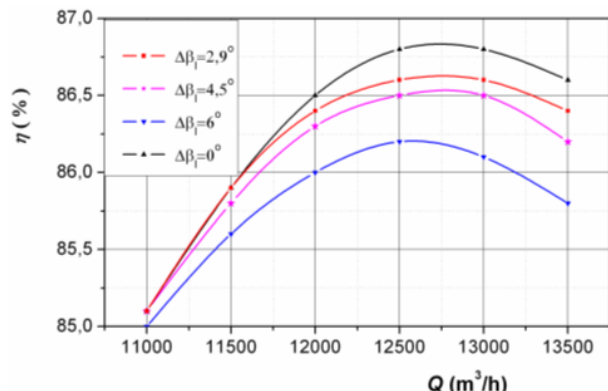

b) $\beta_{1 l}=\beta_{2 l}=\beta_{t}-\Delta \beta_{l}$

Fig. 10 The influence of curvature on the efficiency, $\eta(Q)$

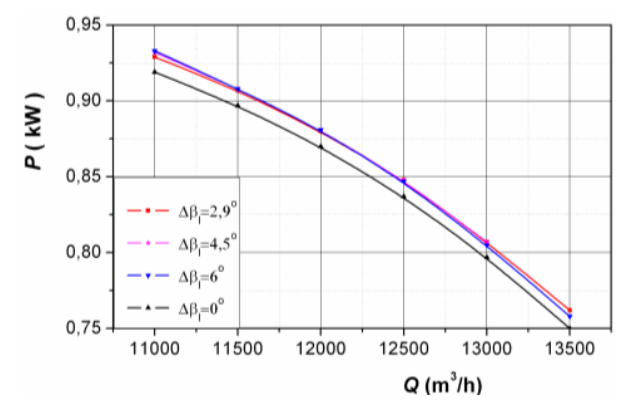

a) $\beta_{1 l}=\beta_{2 l}=\beta_{t}+\Delta \beta_{l}$

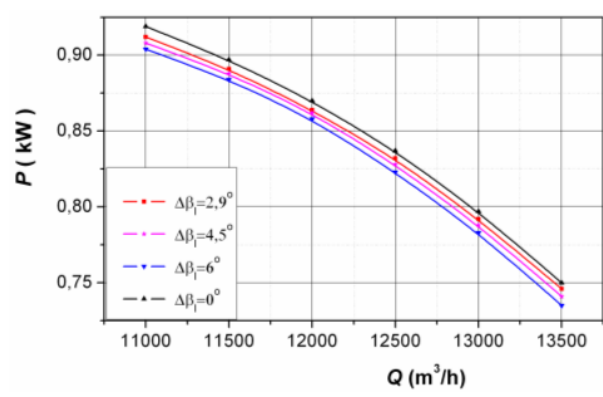

b) $\beta_{1 l}=\beta_{2 l}=\beta_{t}-\Delta \beta_{l}$

Fig. 11 The influence of curvature on the power of the fan, $P(Q)$

Figs. 9-a,b, 10-a,b, 11-a,b show, based on the performed simulation, the performance of the fan with doubly-curved profile of the blades $\left(\beta_{1 l}=\beta_{2 l}=\beta_{t} \pm \Delta \beta_{l}\right)$ and corresponding characteristics of the fan design with the straight profile of the blades $\left(\beta_{1 l}=\beta_{2 l}=\beta_{t}\right.$, $\Delta \beta_{l}=0$ ), for comparison. 


\subsubsection{Analysis of the results obtained for the curved profiles, $\beta_{1 l}=\beta_{2 l}>\beta_{t}$}

(Fig. 9-a, 10-a, 11-a)

Increasing the pressure in the fan with blades that have curved profile ends $\beta_{1 l}=\beta_{2 l}$ $>\beta_{t}$ leads to approximately the same value for angles of curvature $\Delta \beta_{l}=2.9^{0}$ and $\Delta \beta_{l}=4^{0}$, which in relation to the fan with the straight profile is higher for about $2 \%$ (Fig. 9-a). For an angle of curvature $\Delta \beta_{l}=6^{0}$ this difference is the smallest.

With the increase in the angle curvature profile the efficiency of the fan slightly falls. (Fig. 9-b). The difference of efficiency was $0.5 \%$ for the highest flow rate $(\mathrm{Q}=13500$ $\mathrm{m}^{3} / \mathrm{h}$ ), and $1 \%$ for the minimum flow, between the impeller with the straight profile of the blades and the curved profile, for $\Delta \beta_{l}=6^{0}$.

Approximately equal power is needed for all the fans with curved blade profiles, for all the angles of curvature, and it is larger than the power of the fan with straight profiles, an average of $1.3 \%$ for the entire range of the simulated flow (Fig. 9-c).

The optimal operating parameters $\left(\eta_{\max }\right)$ of the fan are achieved with the blades having the following profiles:

- $\quad$ straight $\left(\Delta \beta_{l}=0^{0}\right): \Delta p_{t o l}=200 \mathrm{~Pa}, Q=12750 \mathrm{~m}^{3} / \mathrm{h}, P=0.80 \mathrm{~kW}, \eta=0.868$

- curved, $\beta_{1 l}=\beta_{2 l}>\beta_{t}, \Delta \beta_{l}=2.9^{0}: \Delta p_{\text {tot }}=194 \mathrm{~Pa}, Q=13000 \mathrm{~m}^{3} / \mathrm{h}, P=0.807 \mathrm{~kW}, \eta=0.867$

- curved, $\beta_{1 l}=\beta_{2 l}>\beta_{t}, \Delta \beta_{l}=4.5^{0}: \Delta p_{\text {tot }}=193 \mathrm{~Pa}, Q=13000 \mathrm{~m}^{3} / \mathrm{h}, P=0.807 \mathrm{~kW}, \eta=0.865$

- curved, $\beta_{1 l}=\beta_{2 l}>\beta_{t}, \Delta \beta_{l}=6^{0}: \Delta p_{\text {tot }}=192 \mathrm{~Pa}, Q=13000 \mathrm{~m}^{3} / \mathrm{h}, P=0.805 \mathrm{~kW}, \eta=0.862$

The optimal operating parameters of the fan with curved profiles $\beta_{1 l}=\beta_{2 l}>\beta_{t}$ are obtained at a higher flow rate in relation to the blades of the straight profile by about $2 \%$.

\subsubsection{Analysis of the results obtained for the curved profiles, $\beta_{1 l}=\beta_{2 l}<\beta_{t}$} (Fig. 9-b, 10-b, 11-b)

With the decrease of the leading and trailing angle profile of the blades the fan pressure decreases compared to the straight profiles for the entire range of simulation. For the angle of curvature $\Delta \beta_{l}=6^{0}$ the reduction of the fan pressure is about $2.5 \%$, for the angle of curvature $\Delta \beta_{l}=4.5^{\circ}$ the reduction is about $1.5 \%$, and for the angle of curvature $\Delta \beta_{l}=2.9^{0}$ the reduction is approximately $1 \%$ (Fig. 9-b).

The efficiency throughout the range of the simulated flow rate slightly decreases with the increasing angle of curvature (Fig. 10-b), for the angle of curvature $\Delta \beta_{l}=6^{0}$ the reduction of efficiency is about $0.7 \%$ for the calculated flow $\left(\mathrm{Q}=13000 \mathrm{~m}^{3} / \mathrm{h}\right)$.

With the increase in the angles of curvature the fan power is reduced and it is less than the power of the fan blades with the straight profiles. For the angle of curvature $\Delta \beta_{l}=6^{0}$ the power reduction is approximately $2 \%$ in the whole range of the simulation (Fig. 11-b).

The optimal operating parameters $\left(\eta_{\max }\right)$ of the fan are achieved with the blades having the following profiles:

- straight, $\beta_{1 l}=\beta_{2 l}=\beta_{t}\left(\Delta \beta_{l}=0\right): \Delta p_{t o t}=200 \mathrm{~Pa}, Q=12750 \mathrm{~m}^{3} / \mathrm{h}, P=0.80 \mathrm{~kW}, \eta=0.868$

- curved, $\beta_{1 l}=\beta_{2 l}<\beta_{t}, \Delta \beta_{l}=2.9^{0}: \Delta p_{\text {tot }}=199 \mathrm{~Pa}, Q=12700 \mathrm{~m}^{3} / \mathrm{h}, P=0.815 \mathrm{~kW}, \eta=0.866$

- curved, $\beta_{1 l}=\beta_{2 l}<\beta_{t}, \Delta \beta_{l}=45^{0}: \Delta p_{\text {tot }}=197 \mathrm{~Pa}, Q=12700 \mathrm{~m}^{3} / \mathrm{h}, P=0.792 \mathrm{~kW}, \eta=0.865$

- curved, $\beta_{1 l}=\beta_{2 l}<\beta_{t}, \Delta \beta_{l}=6^{0}: \Delta p_{\text {tot }}=204 \mathrm{~Pa}, Q=12500 \mathrm{~m}^{3} / \mathrm{h}, P=0.823 \mathrm{~kW}, \eta=0.862$ 


\section{CONCLUSIONS}

On the basis of the conducted numerical simulations and the analysis of the obtained fan characteristics, it can be concluded that the profile curvature influences the performance of the axial reversible fan. The highest growth in the fan pressure is achieved with the blades that have the $\beta_{1 l}=\beta_{2 l}>\beta_{t}$ profile angles, with the angle of curvature $\Delta \beta_{l}=2,9^{0}$ for about $2 \%$ of the value of all the simulated flow. The efficiency in the optimal regime is slightly smaller, by about $0.1 \%$. The smallest increment of pressure and the efficiency of the lowest level are achieved in the fan with the blades that have a curved profile with the $\beta_{1 l}=\beta_{2 l}<\beta_{t}$ angles.

The optimal operating parameters of the fan with curved profiles $\beta_{1 l}=\beta_{2 l}>\beta_{t}\left(\Delta \beta_{l}=2,9^{0}\right)$ are obtained at a higher flow rate in relation to the blades of the straight profile by about $2 \%$. The optimal operating parameters of the fan with curved profiles $\beta_{1 l}=\beta_{2 l}<\beta_{t}$ are achieved at a lower flow rate compared to the blades with straight profiles for about $0.4 \%$.

There is a risk involved and thus care should be taken when introducing a double curve in the profile of the blades. Any change in the curve directly affects the separation of the flow from the blade profiles, which can lead to a drop in the characteristics of the fan.

\section{REFERENCES}

1. Spasić, Ž., 2012, Numerical and Experimental Investigation of the Influence of the Blade Profile Shape on the Reversible Axial Fan Characteristics (in Serbian), PhD Thesis, Faculty of Mechanical Engineering Nis, University of Nis, Nis, $131 \mathrm{p}$.

2. Brusilovskij, I.V., 1978, The aerodynamic schemes and characteristics of the axial fan CAGI, (in Russian), Moscow, 197 p.

3. Krsmanović, LJ., Gajić, A., 2000, Turbomachinery-Fans (in Serbian), Faculty of Mechanical Engineering Belgrade, $289 \mathrm{p}$.

4. Cebeci, T., Platzer, M., Chen, H., Chang, K.-C., Shao J.P., 2005, Analysis of Low-Speed Unsteady Airfoil Flows, Springer, Berlin, $171 \mathrm{p}$

5. Parker, D.E., Simonson, M.R., 1982, Transonic Fan/Compressor Rotor Design Study, Volume III-Final Report, Research Laboratories, Wright-Patterson Air Force Base, Ohio.

6. Eck, B., 1973, Fans-Design and Operation of centrifugal, axial-flow and cross-flow fans, Pergamont Press, Oxford, England, 592 p.

7. Bogdanović-Jovanović, J., Milenković, D., Stamenković, Ž., Spasić, Ž, 2017, Determination of averaged axisymmetric flow surfaces and meridian streamlines in the centrifugal pump using numerical simulation results, Facta Universitatis-Series Mechanical Engineering, 15(3), pp. 479-493.

8. Huang, C.H., Gau, C.W., 2012, An optimal design for axial-flow fan blade: theoretical and experimental studies, Journal of Mechanical Science and Technology 26(2), pp. 427-436.

9. Lin, S.C., Shen, M.C., Tso, H.R., Yen, H.C., Chen, Y.C., 2017, Numerical and experimental study on enhancing performance of the stand fan, Applied Sciences, 7(3), 267; doi:10.3390/app7030267.

10. Bamberger, K., Carolus, T., 2017, The development, application, and validation of a quick optimization method for the class of axial fans, ASME Journal of Turbomachinery, 139, p. 111001.

11. Ferziger, J. H., Perić, M., 2002, Computational Methods for Fluid Dynamics, Springer-Verlag Berlin Heidelberg NewYork, 423 p.

12. Bogdanović, B., Bogdanović-Jovanović, J., Spasić, Ž., 2009, Reversible axial fan with blades created of slightly distorted panel profiles, Facta Univesitatis-Series Mechanical Engineering, 7(1), pp 23-36.

13. Spasić, Ž., Milanović, S., Šušteršič, V., Nikolić, B., 2012, Low-pressure reversible axial fan with straight profile blades and relatively high efficiency, Thermal Science, 16(2), pp. S593-S603.

14. Ilikan, N,A., Ayder E., 2015, Influence of the sweep stacking on the performance of an axial fan, ASME Journal of Turbomachinery, 137, p. 0610041.

15. Wallis, A.R., 1983, Axial Flow Fans and Ducts, John Wiey\&Sons, New Yorker, 444 p.

16. Elhadi, E., Kegi, W., 2002, Simulation of vortex flows in axial flow fan using computational fluid dynamics, Pakistan Journal of Information and Technology, 1(3), pp 242-249. 